\title{
Complex lipids
}

\author{
Jean-Marie Saudubray • Matthias R. Baumgartner • \\ Ronald Wanders
}

Published online: 12 November 2014

(C) SSIEM 2014

In a review published in this journal in 2012, 14 inborn errors of metabolism (IEM) related to defects in biosynthesis and remodeling of phospholipids, sphingolipids and fatty acids were delineated and a new field was born (Lamari et al. 2013). Following the rapid expansion of this group of disorders and the elucidation of numerous cellular roles played by the affected molecules, a small group of IEM specialists met in Paris on June 14-15, 2013, to discuss the diagnostic approach and classification of IEM affecting the synthesis and remodeling of complex lipids.

Clinical phenotypes associated with these defects are pleiotropic, appearing to affect all organs and systems (including the central and peripheral nervous system, muscle, eye, skin, bone and cartilage, liver, kidney and immune system). However, the relatively few patients identified to date hamper

\section{J.-M. Saudubray $(\square)$}

Bioclinic and Genetic Unit of Neurometabolic Diseases,

Salpêtrière Hospital, (APHP), 75013 Paris, France

e-mail: jmsaudubray@orange.fr

J.-M. Saudubray

22 Rue Juliette Lamber, 75017 Paris, France

M. R. Baumgartner

Division of Metabolism, University Children's Hospital,

Steinwiesstrasse 75, CH-8032 Zürich, Switzerland

e-mail: Matthias.Baumgartner@kispi.uzh.ch

R. Wanders

Laboratory Genetic Metabolic Diseases Academic Medical Center,

University Hospital Amsterdam, Meibergdreef 9,

1105 AZ Amsterdam, The Netherlands

e-mail: r.j.wanders@amc.uva.nl clinical recognition of these disorders and genotypephenotype correlation.

This special issue, which is predominantly based on presentations during the workshop, is an attempt to summarize the manifestations of more than 100 genetic defects with primary or secondary consequences on complex lipids and fatty acid synthesis and remodeling. Owing to the large number of different diseases and their heterogeneity, it is inevitable that the topic is extremely demanding. Precise definition of the entity of 'complex lipid disorders' remains challenging since it involves many different molecules (triglycerides, phospholipids, phosphatidylinositol, plasmalogens, sphingolipids, cholesterol and other isoprenoids, glycolipids, eicosanoids and complex long and very long chain fatty acids). Furthermore, the involvement of several cellular compartments, and the continuous remodelling of membranes, means that this new group of disorders challenges our concept of catabolic versus anabolic disorders and our current classification of IEM which is still largely based on cellular organelles (mitochondrial, lysosomal, peroxisomal disorders).

While most of these new disorders have been identified by next generation sequencing, of the new field of lipidomics offers additional opportunities to decipher their pathophysiology and to identify therapeutic targets.

This special issue intends to stimulate the field.

\section{References}

Lamari F, Mochel F, Sedel F, Saudubray JM (2013) Disorders of phospholipids, sphingolipids and fatty acids biosynthesis: toward a new category of inherited metabolic diseases. J Inherit Metab Dis 36(3): $411-425$ 